\title{
Implementing Sparse Sub-Sampling Methods for Low-Dose/High Speed STEM
}

Nigel D. Browning ${ }^{1}$ Andrew Stevens ${ }^{2,3}$, Libor Kovarik ${ }^{4}$, Andrey Liyu ${ }^{4}$, B. Layla Mehdi ${ }^{1}$, Hao Yang ${ }^{5}$ Michael E. Gehm ${ }^{3}$, Bryan Stanfill ${ }^{6}$, Sarah Reehl ${ }^{6}$, Lisa Bramer ${ }^{6}$

${ }^{1}$ Physical Sciences \& Engineering, University of Liverpool, Liverpool, L69 3GQ, UK

${ }^{2}$ OptimalSensing, Southlake, Texas 76092, USA

${ }^{3}$ Electrical \& Computer Engineering, Duke University, Durham, North Carolina 27708, USA

${ }^{4}$ Environmental Molecular Sciences Laboratory, PNNL, Richland, WA 99352, USA

${ }^{5}$ Lawrence Berkeley National Laboratory, Berkeley, California 94720, USA

${ }^{6}$ National Security Directorate, PNNL, Richland, WA 99352, USA

In many practical applications of high-resolution scanning transmission electron microscopy (STEM), the resolution obtainable in an image is determined solely by the stability of the sample. This is particularly true in the era of aberration corrected STEM where the dose on the sample for the highest resolution images can easily exceed $10^{5}$ electrons/ $\AA^{2}$ during routine operation. This dose sensitivity presents extreme constraints on applications such as 3-D imaging, spectroscopic imaging and in-situ experiments, where prolonged exposure to the beam can result in damage occurring before the experiment can be completed. To ensure that observations are free from damage artifacts, it is therefore essential that images are acquired under conditions where the maximum information can be extracted from the minimum amount of electron dose. Traditionally, low-dose imaging is performed by either acquiring images faster or changing the emission conditions of the gun. However, when there is a change in the emission the microscope alignment changes and it is not always possible to run the automated corrector alignments in low-dose mode to obtain the highest resolution images. Technological limitations are also associated with obtaining images faster; in the case of the TEM lowdose imaging requirements exceed the framerate of the camera, while in STEM the hysteresis in the scan coils limits the overall scanning speed that can be obtained. To overcome these limitations, image reconstruction methods such as compressive sensing and in-painting [1,2] can be used to reduce the number of read-outs/pixels in the STEM images/movies and lower the overall electron dose while at the same time increasing the speed of the image and reducing the overall size of the dataset acquired $[3,4]$.

The acquisition of sub-sampled STEM images can be achieved through an external scan generator that randomly hops between lines of the scan at a rate designed to minimize the effects of scan hysteresis [5]. For a highly sub-sampled image ( $<10 \%$ of the pixels) the raw image itself is unrecognizable (Figure 1$)$. However, by using the inpainting approach an atomic resolution image can be reconstructed from such an acquisition. It is important to note that all low-dose images are sub-sampled in some way - as the dose is decreased the number of electrons reaching the detector is decreased and some pixels will not receive scattered electrons. This conventional low dose method still samples all the pixels, even the ones that have no electrons, and the image can be reconstructed using the same inpainting algorithm. However, when we deliberately sub-sample the image using a higher electron dose in the sampled pixels (giving an overall equivalent dose to the sample as there are fewer pixels) we increase the image acquisition time to achieve the same high resolution. If the dose is further fractionated in a way that we sub-sample to form a basic reconstructed image and then use that reconstruction to fill in the gaps in the data - termed here as adaptive sub-sampling - we can have the same increase in speed but now also increase the resolution of the image for the same dose (Figure 1). The practical application of this method to study beam sensitive materials is shown in Figure 2, where the nodal distribution in a metal 
organic framework (MOF) is clearly observed at high resolution. The practical aspects of this subsampling approach and the potential for future developments will be discussed in this presentation [7].

\section{References:}

[1] E. J.Candès, J. Romberg, T. Tao, Inform. Theory IEEE Trans. 52, 489-509 (2006)

[2] D. L. Donoho, Inform. Theory IEEE Trans. 52, 1289-1306 (2006)

[3] A. Stevens, H. Yang. L. Carin, I. Arslan and N. D. Browning, Microscopy 63, 41-51 (2014)

[4] A. Stevens, L. Kovarik, X. Yuan, L. Carin, N. D. Browning, ASCI 1, 10 (2015)

[5] L. Kovarik, A. Stevens, A. Liyu, N. D. Browning, Applied Physics Letters 109, 164102 (2016)

[6] A. Stevens, L. Luzi, H. Yang, L. Kovarik, B. L. Mehdi, A. Liyu, M. E. Gehm, N. D. Browning, Applied Physics Letters 112, 043104 (2018)

[7] This work was supported in part by the Chemical Imaging Initiative (CII) and the Analytics in Motion (AIM) Initiative, Laboratory Directed Research and Development (LDRD) Programs at the Pacific Northwest National Laboratory (PNNL). PNNL is a multi-program national laboratory operated by Battelle for the U.S. Department of Energy (DOE) under Contract DE-AC05-76RL01830. A portion of the research was performed in the Environmental Molecular Sciences Laboratory (EMSL), a national scientific user facility sponsored by the DOEs Office of Biological and Environmental Research.
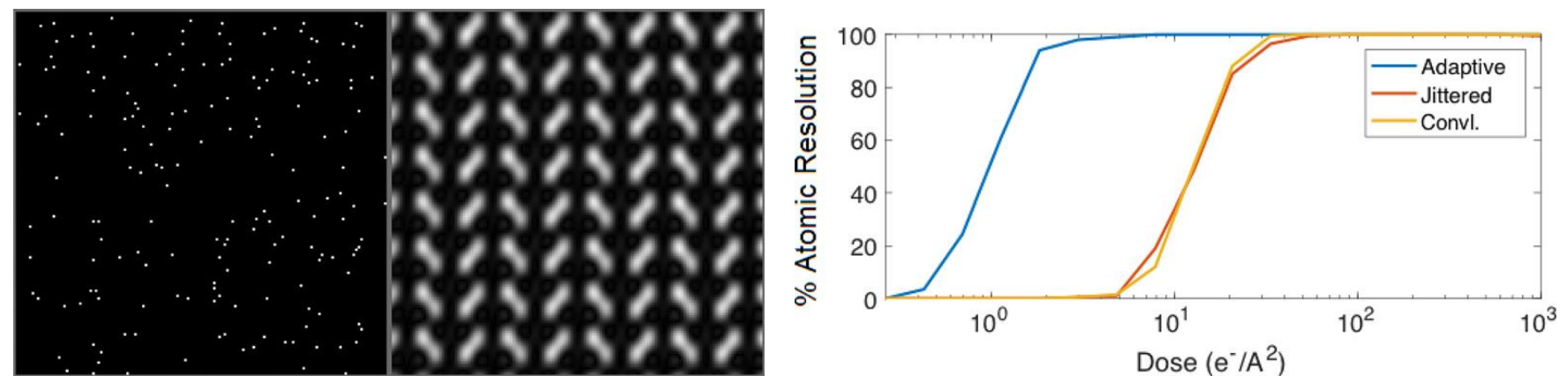

Figure 1: (a) Sub-sampled image of ZnSe, inpainted to a full atomic resolution image (b). (c) The phase transition curve for recovering a full atomic resolution image of $\mathrm{ZnSe}$ using different reconstruction approaches. Adaptive sub-sampling achieves higher resolution, faster and for lower dose.

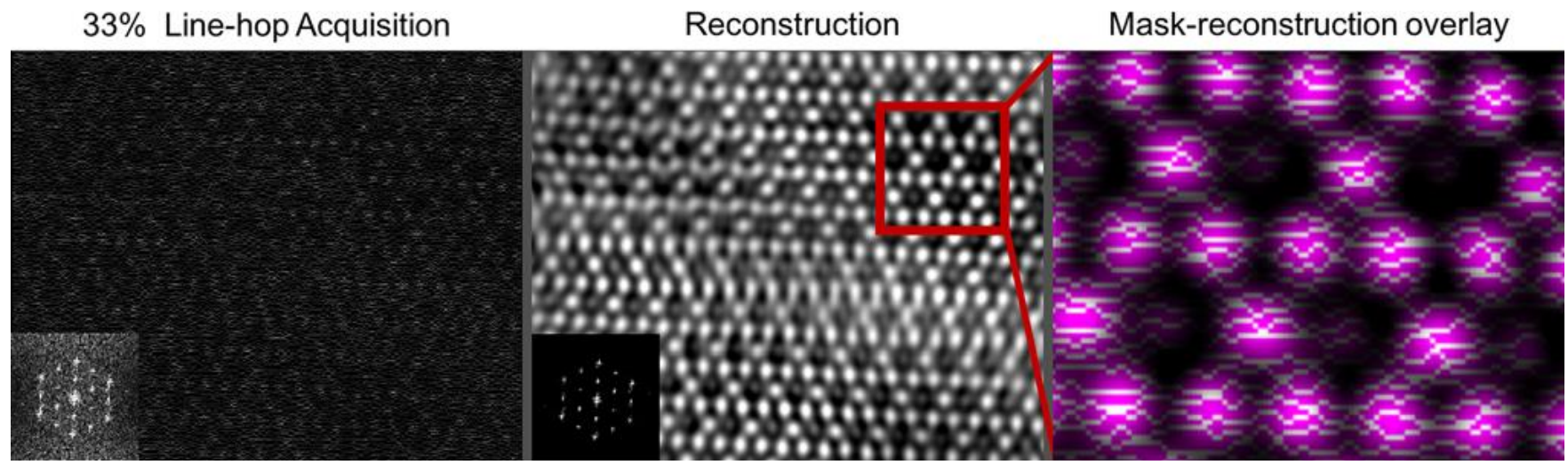

Figure 2: Reconstruction of a sub-sampled line-hop acquisition from an NU-1000 (Zr6Ox) MOF. The distortions are caused by synthesis/preparation. The left panel shows a magnified overlay of the scanlines (white) and the nodes (purple). 